\title{
Developing a national sentinel surveillance system for antimicrobial resistant Neisseria gonorrhoeae in Ireland
}

Aoife Colgan ${ }^{1,2}$, Mary Kelleher ${ }^{2}$, Sinéad Saab², Lisa Rose², Gillian Cullen¹, Stephen Murchan ${ }^{1}$, Brendan

Crowley², Derval Igoe ${ }^{1}$

\section{Background}

3 national surveillance systems for gonorrhoea antimicrobial resistance (GC AMR) surveillance in Ireland:

- $\quad$ Euro-GASP

- HL-AziR

- Treatment failure

Participation in Euro-GASP is via decentralised testing in the Central Pathology Laboratory in St. James's Hospital (SJH). $\mathrm{SJH}$ was designated the Interim National Gonococcal Reference Laboratory (INGCRL) in 2017 to improve national GC AMR surveillance.

\section{Methods}

From 2010 - 2016, isolates originated from two main STI clinics. In 2017, isolates could be submitted from external laboratories with other clinical sources. However, only a small numbers of isolates ( $n=11)$ submitted to Euro-GASP in 2017 came from external laboratories.

All isolates were tested for susceptibility to:

- Cefixime

- Ceftriaxone

- Azithromycin MIC strip

- Ciprofloxacin

And for production of $\beta$-lactamase - Nitrocefin

Epidemiological data were collected on all patients using a combination of electronic patient records, enhanced surveillance forms and the Computerised Infectious Diseases Reporting (CIDR) tool.

\section{Patient Demographics}

775 isolates were reported to Euro-GASP, 2010 - 2017

( $7 \%$ of total national notifications reported during that time):

\begin{tabular}{|l|l|l|}
\hline Variable & $\begin{array}{l}\text { Data } \\
\text { available }\end{array}$ & Percent (where known) \\
\hline Sex & $100 \%$ & $\begin{array}{l}92 \% \text { males } \\
8 \% \text { females }\end{array}$ \\
\hline Age & $100 \%$ & $\begin{array}{l}\text { Range: } 15-65 \text { years } \\
\text { Median (male): } 28\end{array}$ \\
\hline $\begin{array}{l}\text { Median (female): } 23 \\
\text { Region of birth }\end{array}$ & $75 \%$ & $\begin{array}{l}\text { Ireland: } 60 \% \\
\text { Latin America: } 18 \% \\
\text { Western Europe: } 10 \% \\
\text { Central/Eastern Europe: } 8 \% \\
\text { Other regions: } 4 \%\end{array}$ \\
\hline $\begin{array}{l}\text { Mode of } \\
\text { transmission }\end{array}$ & $92 \%$ & $\begin{array}{l}\text { MSM: } 78 \% \\
\text { Heterosexual male: } 14 \% \\
\text { Heterosexual female: } 9 \%\end{array}$ \\
\hline $\begin{array}{l}\text { HIV status } \\
\text { Previous }\end{array}$ & $84 \%$ & $\begin{array}{l}12 \% \text { HIV positive } \\
88 \% \text { HIV negative }\end{array}$ \\
\hline $\begin{array}{l}\text { gonorrhoea } \\
\text { Concurrent } \\
\text { chlamydia }\end{array}$ & $88 \%$ & $\begin{array}{l}21 \% \text { had previous } \\
\text { gonorrhoea infection }\end{array}$ \\
\hline $\begin{array}{l}\text { Compliance with } \\
\text { dual therapy }\end{array}$ & $37 \%$ & $\begin{array}{l}15 \% \text { had concurrent } \\
\text { chlamydia infection } \\
89 \% \text { received recommended } \\
\text { dual therapy }\end{array}$ \\
\hline & $94 \%$ &
\end{tabular}

\section{Antimicrobial Resistance, 2010 - 2017}

- $99 \%$ of isolates were susceptible to cefixime.

There has been drift towards lower MICs since ceftriaxone was introduced as treatment for GC.
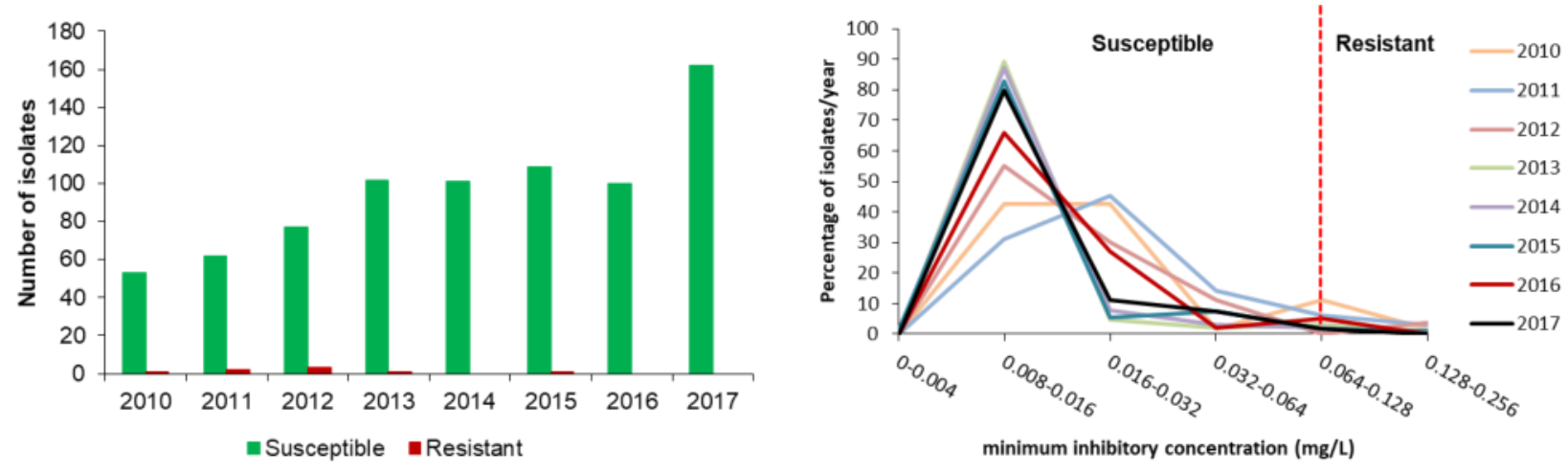

- Only 1 reported isolate was resistant to ceftriaxone.

There has been drift towards higher MICs in recent years.
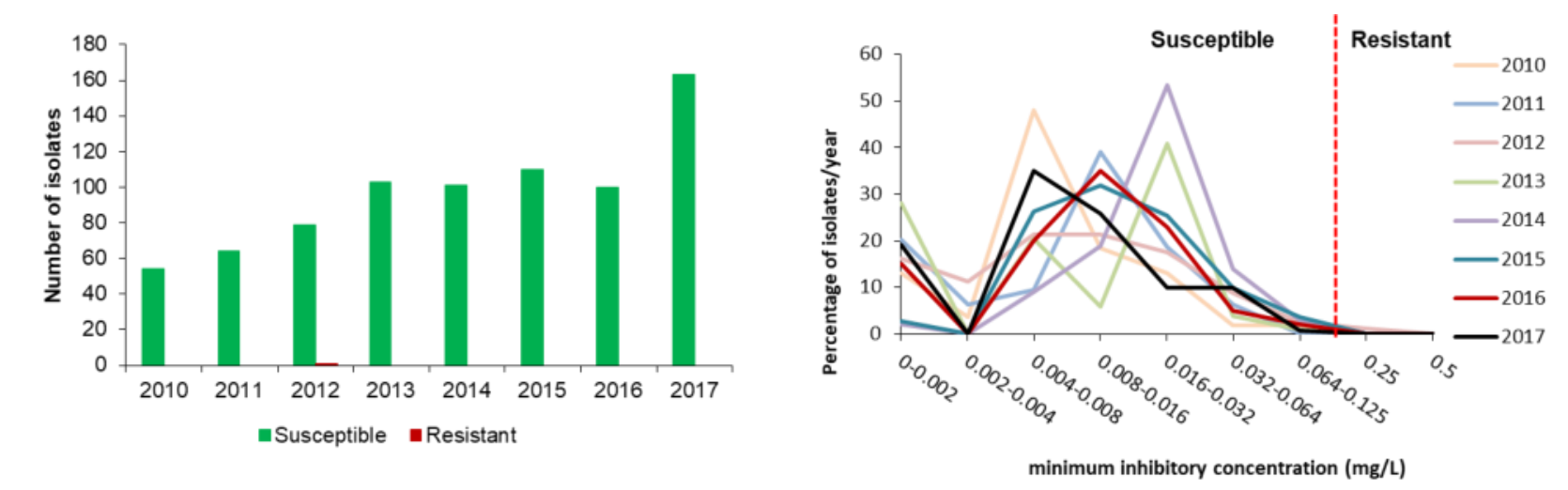

- $13 \%$ of isolates were resistant to azithromycin.

The proportion resistant has been $>10 \%$ since 2014 .
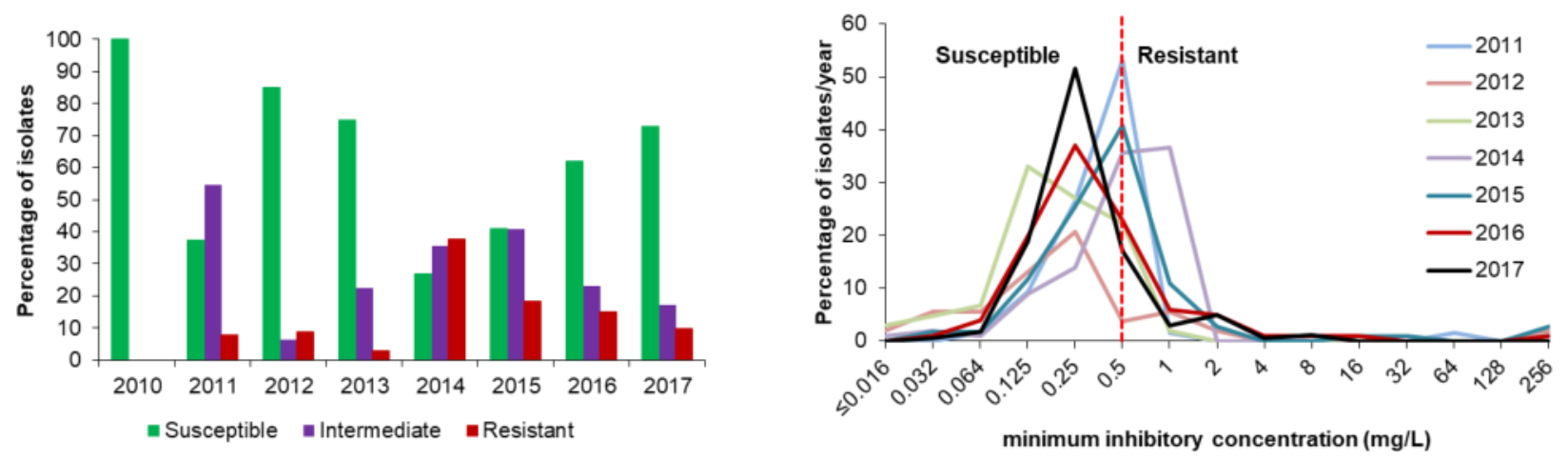

- No isolates with reduced susceptibility to ceftriaxone were also resistant to azithromycin

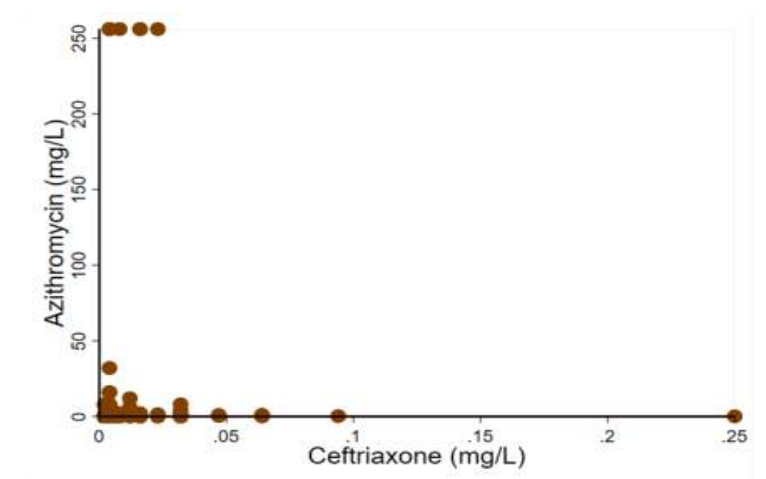

- $37 \%$ of isolates were resistant to ciprofloxacin.

- $9 \%$ of isolates produced $\beta$-lactamase.

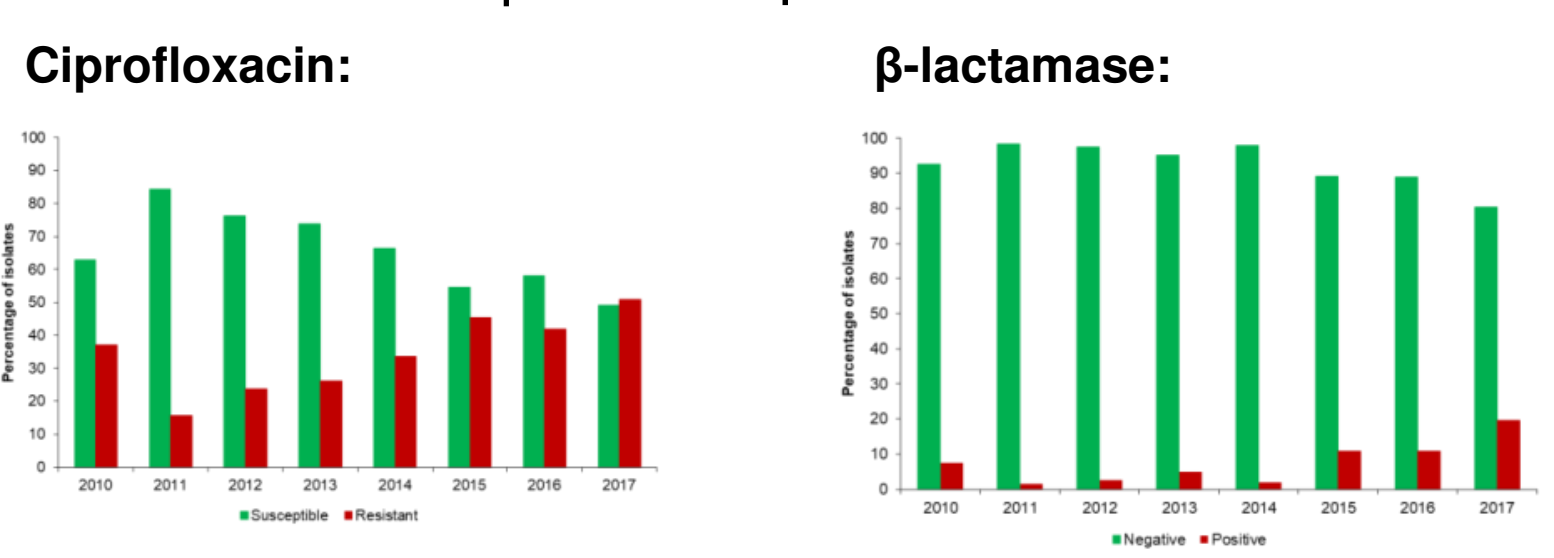

National sentinel surveillance: Conclusions Designation of the INGCRL in 2017 provides an opportunity for the Irish Euro-GASP dataset to become more nationally representative.

Laboratory and surveillance mechanisms have been established that will aid in a move towards a more nationally representative dataset in 2018, and a national sentinel surveillance system for GC AMR.

${ }^{1}$ Health Protection Surveillance Centre, 25-27 Middle Gardiner St., Dublin 1
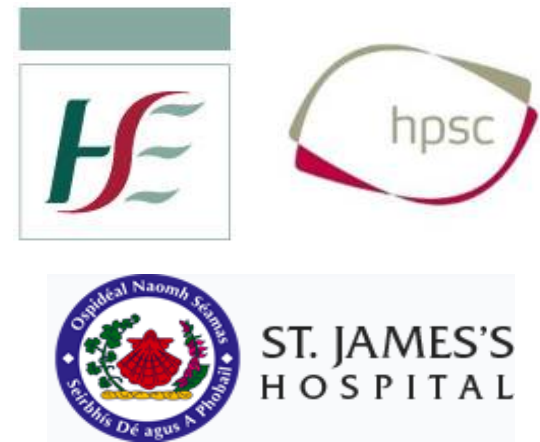